\title{
The best radionuclide for radioimmunotherapy of small tumors: beta- or alpha-emitter?
}

\author{
Jacques Barbet $\cdot$ Jean-François Chatal
}

Published online: 16 December 2010

(C) Springer-Verlag 2010

The efficacy of locoregional injection of monoclonal antibodies labeled with alpha-emitting radionuclides in solid tumors has been demonstrated in some preclinical models and clinical settings. The Munich group has published several promising studies in gastric cancer with the $\mathrm{d} 9 \mathrm{Mab}$ that recognizes the mutant d9-E-cadherin [1] and in bladder cancer with an anti-EGFR antibody [2], both labeled with bismuth-213. Similarly, Lars Jacobson and coworkers have treated ovarian cancer with a ${ }^{211}$ At-labeled $\mathrm{F}\left(\mathrm{ab}^{\prime}\right)_{2}$ antibody fragment recognizing a cell surface antigen expressed by human epithelial ovarian cancers [3]. Michael Zalutsky and coworkers have treated recurrent brain tumors by injecting ${ }^{211}$ At-labeled anti-tenascin antibody in the tumor resection cavity, obtaining survival improvement compared with a historical control group of patients [4]. In all cases, efficient treatment was possible with acceptable toxicity for a precisely optimized injected activity because most of the activity remained at the site of injection.

The locoregional route is preferable for these short-lived radionuclides when targeted by antibodies because using the intravenous route results in significant radionuclide decay before the antibody has reached its target. Smaller carrier molecules, such as diabodies, peptides, or pretargeted haptens, may be more appropriate in that case. For hematological diseases, systemic administration is needed and appears feasible.

A common observation is that anti-tumor efficacy decreases with tumor burden [1,3]. Toxicity of alphaemitting radionuclides does indeed prevent the injection of high activities, which means that the targeting procedures

J. Barbet $\cdot$ J.-F. Chatal $(\bowtie)$

GIP Arronax, Nantes University,

Nantes, France

e-mail: chatal@arronax-nantes.fr are not yet optimal. Then the number of injected radioactive atoms is not so large, and in case of large tumors it may be of the same order of magnitude as the number of tumor cells, or even lower: in a study published in this issue of the European Journal of Nuclear Medicine and Molecular Imaging, Seidl et al.[5], calculated that $1.85 \mathrm{MBq}$ correspond to only $7.4 \times 10^{9}$ bismuth-213 atoms. Only a fraction of these atoms will become bound to tumor cells and several bismuth atoms are needed to kill one tumor cell, so assuming $10 \%$ binding and 10 atoms needed, only 74 million cells could be killed, which corresponds to a tumor mass of about $74 \mathrm{mg}$, usual in preclinical therapeutic studies.

However, this is certainly not the only reason for such a tumor size effect. In the study by Seidl et al., treatment of locally disseminated gastric cancer in nude mice by intraperitoneal injection of ${ }^{177}$ Lu-labeled antibody significantly prolonged mouse survival, but therapy at day 1 after tumor cell inoculation was more effective than on day 8 . The best results were obtained with $7.4 \mathrm{MBq}$ of ${ }^{177} \mathrm{Lu}-\mathrm{d} 9 \mathrm{MAb}$, with $90 \%$ of animals surviving longer than 250 days. The authors conclude that the ${ }^{177}$ Lu-labeled antibody has anti-tumor efficacy similar to that of the ${ }^{213} \mathrm{Bi}$-labeled antibody, which showed optimal efficacy with acceptable toxicity at a dose of $1.85 \mathrm{MBq}$. They also discuss the specificity of the treatment. The non-specific antibody labeled with lutetium-177 showed some efficacy at higher doses, whereas the bismuth-213 nonspecific antibody showed very little antitumor activity. This probably results from the millimeter range of the beta particles emitted by lutetium-177 and from the relatively long residence time of the antibody in the peritoneum cavity.

Given the large difference in half-life (46 $\mathrm{min}$ for bismuth-213 and 6.71 days for lutetium-177) with the similar specific activities, the authors remark that 825 times more ${ }^{177}$ Lu-labeled antibodies than ${ }^{213}$ Bi-labeled antibodies 
would have to be injected for a similar therapeutic effect. They conclude that one $\alpha$-particle is as effective as 1,000 $\beta$-particles, which is an oversimplification, since most bismuth-213 atoms probably decay in the peritoneal cavity whereas a significant fraction of lutetium-177 escapes before decay in the cavity. More interestingly, they also remark that lutetium-177 and bismuth-213 result in very different cumulative activities: $42.2 \mathrm{MBq} \cdot \mathrm{h}$ for $1.85 \mathrm{MBq}$ of lutetium-177 over $24 \mathrm{~h}$ and only $2.05 \mathrm{MBq} \cdot \mathrm{h}$ for the same bismuth-213 activity. The comparison should have been pushed further in terms of tumor absorbed doses, since decay of one lutetium-177 atom delivers much less energy than that of a bismuth-213 atom $(0.2$ and $8.3 \mathrm{MeV}$, respectively).

In terms of toxicity, the ${ }^{177} \mathrm{Lu}$-labeled antibody showed myelotoxicity, which probably results from the physiological transfer of unbound antibody from the peritoneal cavity to the circulation. The process is relatively slow (maximum blood activity is observed 1 day after injection) and is certainly negligible for bismuth-213. By contrast, the ${ }^{213} \mathrm{Bi}$-labeled antibody was found nephrotoxic at high doses. This would imply that some activity quickly leaks out of the peritoneum, probably in the form of free bismuth, which would then stick into the kidneys. Quantifying this fraction is certainly very difficult with such a short half-life. Whether this nephrotoxicity could be decreased by further reduction of free bismuth in the preparation or by using more stable chelation techniques is an unresolved issue.

Quite unusually some oncogenic effects have been reported in this study with lutetium-177 and not with bismuth-213; these included lymphoblastic lymphoma, plasmocytoma, and hepatocarcinoma developed in $10 \%$ of mice 120-145 days after injection of moderate activity of 7.4 and $14.8 \mathrm{MBq}$ of lutetium-177. These effects were related to a long half-life of lutetium-177 activity and the slow excretion of lutetium-177 immunoconjugates.

However, this explanation is not entirely convincing. In other preclinical studies performed with antibodies labeled with lutetium-177 no such oncogenic effect has been reported. As such effects may occur only in the long-term, such as 120-145 days after injection in the present study, the absence of previous observation of oncogenic effects could be related to excessively short follow-up times, not extending beyond a few weeks in the majority of previous studies. As an example, the maximum follow-up time was respectively 77 and 102 days in two studies performed with antibodies labeled with lutetium-177 [6, 7]. However, in one study performed with a ${ }^{177} \mathrm{Lu}$-labeled anti-Lewis Y antibody the maximum follow-up time was 150 days after injection of an activity of $12.95 \mathrm{MBq}$, comparable to that of the present study, and no oncogenic effect was observed [8]. Furthermore, a lot of preclinical studies have been carried out with antibodies labeled with iodine-131, which has a half-life longer than that of lutetium-177, and oncogenic effects have never been reported. The authors of the present study relate the oncogenic effects to a long residence time of ${ }^{177} \mathrm{Lu}$-immunoconjugate in the blood, with blood levels decreasing from $15 \% \mathrm{ID} / \mathrm{g}$ on day 3 to $9.7 \%$ on day 7 , but the same blood levels have been reported with a ${ }^{131}$ I-labeled antibody to carcinoembryonic antigen and no oncogenic effects have been observed after a maximum follow-up time of 142 days [9]. Thus there is no clear explanation for the oncogenic effects observed in the present study, which has never been previously reported in multiple studies carried out with a lot of antibodies labeled using radionuclides with half-lives in the same range or longer than that of lutetium-177. Other causes not related to irradiation, such as the mouse strain used or the antibody by itself, could be considered.

In conclusion, there is no clear-cut explanation for the oncogenic effects observed in this study. It could only be recommended for future studies to extend the follow-up time to more than 200 days following the therapeutic injection of radioimmunoconjugates in order to look for possible long-term oncogenic effects.

Even if extrapolation of preclinical results to the clinical situation should be quite cautious, it is important to emphasize that the types of oncogenic effects observed in the present preclinical study have never been reported in a high number of clinical studies performed during the last 50 years. In a report on 47,712 patients with benign and malignant diseases of the thyroid and administered with therapeutic or diagnostic doses of iodine-131 no increased incidence of oncogenic effects, especially leukemia, has been reported after 5 years' followup. However, some rare cases of leukemia have been reported after high cumulative doses of more than $29.6 \mathrm{GBq}(800 \mathrm{mCi})$ of iodine-131 [10]. With regard to radioimmunotherapy a cumulative incidence of treatment-related myelodysplastic syndromes and acute myeloid leukemia after ${ }^{131}$ I-labeledtositumomab injection in 995 previously heavily pretreated patients with non-Hodgkin lymphoma was $0.8 \%$ and $5 \%$ respectively at 2 and 5 years [10]. With regard to peptide receptor radionuclide therapy, the only oncogenic effect observed in 504 patients with gastroenteropancreatic neuroendocrine tumors treated with $\left[{ }^{177} \mathrm{Lu}-\mathrm{DOTA}{ }^{0}, \mathrm{Tyr}^{3}\right]$ octreotate between 2000 and 2006 was myelodysplastic syndrome in four patients $(0.8 \%)$ [11].

Thus it appears that the oncogenic effects observed in thousands of patients after radionuclide therapy using different radionuclides including iodine-131, lutetium-177 and yttrium-90 are limited to treatment-related myelodysplastic syndromes and acute myeloid leukemia. Oncogenic effects observed in the present preclinical study have never been reported in patients. Their origin thus remains unclear and could be related to various parameters including the mouse strain, the antibody by itself, or murine specificity. 
Altogether, these preclinical studies on gastric cancer disseminated in the peritoneal cavity in mice show that lutetium-177-labeled antibody does not appear to have better anti-tumor activity than the same antibody labeled with bismuth-213 in the same preclinical model and that the toxicity pattern seems better with the alpha-emitting radionuclide. Obviously, further comparisons in other models are urgently needed.

\section{References}

1. Beck R, Seidl C, Pfost B, Morgenstern A, Bruchertseifer F, Baum $\mathrm{H}$, et al. ${ }^{213} \mathrm{Bi}$-radioimmunotherapy defeats early-stage disseminated gastric cancer in nude mice. Cancer Sci. 2007;98:121522.

2. Pfost B, Seidl C, Autenrieth M, Saur D, Bruchertseifer F, Morgenstern A, et al. Intravesical alpha-radioimmunotherapy with ${ }^{213} \mathrm{Bi}$-anti-EGFR-mAb defeats human bladder carcinoma in xenografted nude mice. J Nucl Med. 2009;50:1700-8.

3. Elgqvist J, Andersson H, Bäck T, et al. Alpha-radioimmunotherapy of intraperitoneally growing OVCAR-3 tumors of variable dimensions: outcome related to measured tumor size and mean absorbed dose. J Nucl Med. 2006;47:1342-50.

4. Zalutsky MR, Reardon DA, Akabani G, Coleman RE, Friedman AH, Friedman HS, et al. Clinical experience with alpha-particle-emitting ${ }^{211} \mathrm{At}$ : treatment of recurrent brain tumor patients with ${ }^{211}$ At-labeled chimeric antitenascin monoclonal antibody 81C6. J Nucl Med. 2008;49:30-8.
5. Seidl C, Zöckler C, Beck R, Quintanilla-Martinez L, Bruchertseifer F, Senekowitsch-Schmidtke R. ${ }^{177}$ Lu-immunotherapy of experimental peritoneal carcinomatosis shows comparable effectiveness to ${ }^{213} \mathrm{Bi}$-immunotherapy, but causes toxicity not observed with ${ }^{213} \mathrm{Bi}$. Eur J Nucl Med Mol Imaging 2011. doi:10.1007/ s00259-010-1639-2.

6. Schlom J, Siler K, Milenic DE, Eggensperger D, Colcher D, Miller LS, et al. Monoclonal antibody-based therapy of a human tumor xenograft with a ${ }^{177}$ lutetium-labeled immunoconjugate. Cancer Res. 1991;51:2889-96.

7. Persson M, Gedda L, Lundqvist H, Tolmachev V, Nordgren H, Malmström PU, et al. 177Lu pertuzumab: experimental therapy of HER-2-expressing xenografts. Cancer Res. 2007;67:326-31.

8. Kelly MP, Lee ST, Lee FT, Smyth FE, Davis ID, Brechbiel M, et al. Therapeutic efficacy of ${ }^{177} \mathrm{Lu}-\mathrm{CHX}-\mathrm{A}^{\prime \prime}$-DTPA-hu3S193 radioimmunotherapy in prostate cancer is enhanced by EGRF inhibition or docetaxel chemotherapy. Prostate. 2009;69:92-104.

9. Koppe MJ, Bleichrodt RP, Soede AC, Verhofstad AA, Goldenberg DM, Oyen WJG, et al. Biodistribution and therapeutic efficacy of ${ }^{125 / 131} \mathrm{I}$-, ${ }^{186} \mathrm{Re}-$, ${ }^{88 / 90} \mathrm{Y}$-, or ${ }^{177} \mathrm{Lu}$-labeled monoclonal antibody MN-14 to carcinoembryonic antigen in mice with small peritoneal metastases of colorectal origin. J Nucl Med. 2004;45:1224-32.

10. Bennett JM, Kaminski MS, Leonard JP, Vose JM, Zelenetz AD, Knox SJ, et al. Assessment of treatment-related myelodysplastic syndromes and acute myeloid leukemia in patients with nonHodgkin lymphoma treated with tositumomab and iodine I-131 tositumomab. Blood. 2005;105:4576-82.

11. Kwekkeboom DJ, de Herder WW, Kam BL, van Eijck CH, van Essen M, Kooij PP, et al. Treatment with the radiolabeled somatostatin analog [177Lu-DOTA0, Tyr3]octreotate: toxicity, efficacy, and survival. J Clin Oncol. 2008;26:2124-30. 